\title{
Digestive System Injury
}

National Cancer Institute

\section{Source}

National Cancer Institute. Digestive System Injury. NCI Thesaurus. Code C35237.

Trauma to the gastrointestinal tract or accessory org ans of digestions. 